\title{
The Effect of Lifitegrast on Refractive Accuracy and Symptoms in Dry Eye Patients Undergoing Cataract Surgery
}

This article was published in the following Dove Press journal: Clinical Ophthalmology

John Hovanesian (D) Alice Epitropoulos (D) ${ }^{2}$

Eric D Donnenfeld (D) $^{3}$ Jack T Holladay $\mathbb{B D}^{4}$

'Harvard Eye Associates, Laguna Hills, CA, USA; ${ }^{2}$ Ophthalmic Surgeons \& Consultants of Ohio, Columbus, $\mathrm{OH}$, USA; ${ }^{3}$ Ophthalmic Consultants of Long Island, Garden City, NY, USA; ${ }^{4}$ Baylor College of Medicine, Houston, TX, USA

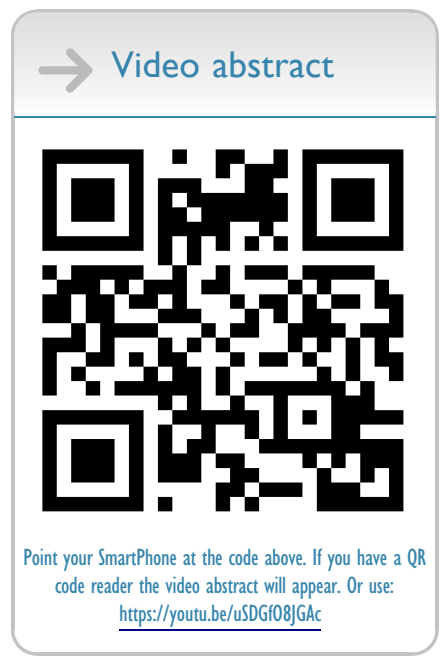

Correspondence: John Hovanesian Harvard Eye Associates, 2440I Calle De La Louisa, Laguna Hills, CA 92653, USA Tel + I 949 95। 2020

$\mathrm{Fax}+19499519244$

Email jhovanesian@harvardeye.com
Purpose: To determine the effect of lifitegrast ophthalmic solution 5\% on improving the tear film, biometry/keratometry, and refractive accuracy for dry eye patients scheduled for cataract surgery.

Patients and Methods: Multicenter, prospective, open-label study of 100 eyes of 100 patients undergoing cataract surgery who had a confirmed diagnosis of dry eye. Patients underwent biometry at baseline and again after a 28-day course of lifitegrast 5\% BID. Primary outcome was an improvement in the accuracy of preoperative anterior corneal power measurements at predicting postoperative spherical equivalent (SE) pre- and post-lifitegrast treatment. Secondary outcomes included changes in dry eye symptoms and corneal staining.

Results: The accuracy of the biometry readings for the achieved refractive SE: within $0.25 \mathrm{D}$ in $47 \%$ and $50 \%$ of eyes before and after the initial lifitegrast treatment, respectively; within 0.5 $\mathrm{D}$ in $71 \%$ and $79 \%$ of eyes before and after the initial lifitegrast treatment; and within $0.75 \mathrm{D}$ in $81 \%$ and $91 \%$ of eyes before and after the initial lifitegrast treatment $(\mathrm{p}<0.04)$.

Conclusion: Lifitegrast 5\% significantly improved preoperative corneal surface measurement accuracy in patients with confirmed dry eye who were scheduled for cataract surgery. Keywords: cataract surgery, dry eye, lifitegrast, higher-order aberrations

\section{Plain Language Summary}

In cataract surgery, the patient's natural lens is replaced with an artificial lens implant that achieves its focusing power based on measurements of the patient's eye taken before surgery. The accuracy of those measurements is important because it determines how well focused the patient's eye will be after surgery. It is difficult to measure the eye accurately in patients who have dry eyes because the surface of the eye is irregular, which causes the cornea to absorb stain during the exam and to have the surface tears break up quickly. Lifitegrast $5 \%$ is an eye drop and the only drug approved by the US Food and Drug Administration to treat both signs and symptoms of dry eye. In this study, we determined whether treatment with lifitegrast 5\% drops for 28 days before surgery improved the smoothness of the surface of the eye and whether this improvement actually led to better lens implant calculations, compared to doing these measurements without lifitegrast treatment. The study, performed on about 100 people, found significant improvement in all outcomes measured.

\section{Introduction}

Cataract surgery is one of the most frequently performed surgeries in the United States with favorable outcomes in about $98 \%$ of cases. ${ }^{1,2}$ Outcomes are dependent upon 
accurate intraocular lens (IOL) calculations, which require axial length, keratometry, and anterior chamber depth. They are also dependent upon a healthy ocular surface, using the proper IOL formula, and a variety of additional measurements of the eye including refraction, pupil size, and effective lens position. ${ }^{3-5}$ Clinical practice guidelines assert biometry is the single most important factor impacting patient outcomes and satisfaction, ${ }^{6,7}$ but, while optical axial length measurements have improved dramatically compared to older ultrasound techniques, the accuracy of corneal power measurements has not meaningfully improved with newer technology. Inaccurate corneal power measurements can result in incorrect IOL power calculations, which in turn can lead to postoperative complications that may need additional surgical correction. ${ }^{8,9}$

A number of factors can impact the accuracy of biometric readings, including ocular surface disease, irregularities in the tear film, and dry eye disease (DED).$^{10-13} \mathrm{An}$ abnormal tear film can lead to inaccurate preoperative measurements impacting the final IOL calculation. ${ }^{13-16}$ Epitropoulos et al found that IOL power calculations can vary as much as $0.5 \mathrm{D}$ in patients with dry eye when those same patients were measured at different visits. ${ }^{14}$

Anterior segment surgeons estimate that about $20 \%$ of their cataract surgery patients have ocular surface disease and/or DED preoperatively. ${ }^{15-17}$ But the PHACO study found closer to $60 \%$ of patients undergoing screening for routine cataract surgery had rapid tear breakup time (TBUT) and approximately $50 \%$ had central corneal staining, both of which are hallmarks of DED. ${ }^{18}$

Numerous treatments for DED are commercially available, ${ }^{19-24}$ but there has been nothing reported on the impact these treatments specifically have on anterior corneal power readings in patients undergoing cataract surgery when compared to outcomes in patients who do not undergo dry eye treatments preoperatively. Lifitegrast $5 \%$ (Xiidra; Novartis, Basel, Switzerland) is a pharmacologic treatment approved for treating both the signs and symptoms of DED. ${ }^{25-30}$ Corneal staining, one of the cardinal signs of DED that is known to reduce the accuracy of keratometry readings, was improved significantly after 4 weeks of treatment in the FDA registration trial of lifitegrast, so it seems an appropriate treatment choice for preparing eyes for cataract surgery biometry. ${ }^{18,27,28,31} \mathrm{In}$ this study, we sought to determine if the refractive accuracy of anterior corneal power readings is improved when lifitegrast is administered preoperatively to patients who are scheduled for cataract surgery and who have confirmed
DED (defined here as a TBUT $\leq 10$ seconds and central or inferior corneal staining as defined by the Oxford Scale).

Higher order aberrations (HOAs) have been linked to patient satisfaction especially when multifocal lenses are implanted, with greater HOAs leading to dissatisfaction with these advanced lenses. Many surgeons with experience in multifocal lens implantation consider HOAs $\leq 0.5$ $\mu$ to be an acceptable upper limit of corneal HOAs when considering a patient for a multifocal lens; this threshold has also been documented in the literature. ${ }^{32,33}$

\section{Patients and Methods}

This was a multicenter, prospective, open-label, study of 100 eyes of 100 patients with planned cataract surgery who had a preoperative diagnosis of dry eye, characterized by central or inferior corneal fluorescein staining and a TBUT $\leq 10$ seconds on slit-lamp examination. All procedures were performed by three surgeons (JAH, EDD, $\mathrm{AE}$ ), and all preoperative measurements were performed using the same diagnostic equipment before and after treatment with lifitegrast. The study is registered on ClinicalTrials.gov (NCT03866629) and adhered to both the Declaration of Helsinki and good clinical practices as defined by the U.S. Food and Drug Administration. Reasonable requests for de-identified patient data relating to the study findings will be available through the corresponding author for 5 years following the publication date.

Patients were excluded if they had previous ocular surgery (intraocular, oculoplastic, corneal, or refractive surgical procedures) performed within the last 3 months or at any time if it would, in the investigator's clinical judgment, interfere with the study's outcome measures. Additional exclusion criteria were ocular inflammation (conjunctivitis, uveitis, iritis, scleritis, episcleritis, or keratitis not related to the qualifying dry eye diagnosis), ocular scarring, dystrophy, or disease that would interfere with ocular surface integrity. Use of an over-the-counter dry eye lubricant or any other dry eye treatment (ie, warm compresses) was not an exclusion factor, but patients were instructed to continue any pre-enrollment treatments unchanged as they added lifitegrast during the study period. Patients provided verbal consent and signed an informed consent document approved by Aspire IRB (Santee, California, USA). Patients were instructed to continue any baseline dry eye treatments they were using. Compliance was assessed during the study by asking patients to report on their use of lifitegrast. Those patients who reported not using the drug, or not using it for the entire prescribed time, were excluded. 
The study was first designed to recruit 200 participants, based on calculations from assumptions about the effect lifitegrast would have on measurement accuracy. As this open-label study progressed, we had achieved statistical significance for HOA improvement after only 34 patients and were nearing significance for our primary outcome measure of refractive accuracy. We then elected to truncate the study to 100 participants. We also modified the protocol to seek an additional set of outcome measures that would come after surgery-determining whether the ocular surface benefits of lifitegrast would continue if it were re-started about 28 days after surgery and continued for four weeks until about postoperative day 56 . These additional outcome measures were added to confirm the value of lifitegrast in maintaining a healthy ocular surface after surgery.

\section{Assessments}

Patients who met the inclusion criteria underwent biometry (including anterior corneal power readings) with an IOL Master 500 or 700 (Zeiss; Dublin, California, USA), corneal topography with a Zeiss Atlas 900 or later topographer, slit lamp examination (including assessing conjunctival hyperemia [Schulze $\left.\operatorname{scale}^{34}\right]$ ), corneal staining (Oxford scores), and TBUT. Patients also completed a Standardized Patient Evaluation of Eye Dryness (SPEED) questionnaire, a validated patient-reported outcome instrument for DED where scores $>10$ are considered abnormal. ${ }^{35}$ Patients were then prescribed a 28-day course of lifitegrast $5 \%$ BID, after which the same biometry measurements were taken. Cataract surgery was scheduled 1 to 3 weeks after completion of the lifitegrast course, with the attendant surgeon choosing an IOL implant power based on the post-lifitegrast biometry reading.

Postoperatively, beginning about 30 days after surgery, patients were instructed to administer a second, 28-day course of lifitegrast $5 \%$ BID after the end of routine postoperative medications (antibiotic, steroid, and non-steroidal antiinflammatory drug). After completion of the second course of lifitegrast (approximately 56 days after cataract surgery), patients completed the SPEED questionnaire, underwent corneal topography, and slit-lamp examination (measuring conjunctival hyperemia, corneal staining, and TBUT; Data on file).

\section{Data Analysis}

The same IOL power calculation formula was used for both sets of measurements for each patient. For ethical reasons, surgeons were permitted to use the formula of their choice. All participating surgeons used the latest generation formulas for accuracy. The predicted final spherical equivalent (SE) refraction was noted for the IOL model and power actually used in surgery. The pre- and post-lifitegrast power predictions were compared to the final SE refraction measured 28 days after surgery to determine the accuracy of each prediction.

Total SPEED questionnaire scores and scores for conjunctival hyperemia, corneal staining, and TBUT were recorded in a database and compared for means and standard deviation; paired t-testing was conducted to test for significance at the $95 \%$ confidence level. Root-mean square higher-order aberrations (HOAs) in the central $6.0 \mathrm{~mm}$ of the cornea were measured and recorded by the Zeiss Atlas topographer (Dublin, California, USA) before and after lifitegrast treatment and compared for each patient. These were also compared with paired t-tests.

\section{Results}

There were 103 patients enrolled; 83 of whom completed the preoperative course of lifitegrast with good compliance. The remaining 20 patients (19.4\%) were excluded for non-compliance. Average age of participants was 71.3 \pm 11.6 years. Fifty four $(52 \%)$ were females.

\section{Refractive Accuracy}

Seventy-three eyes had anterior corneal power measurements performed both before and after 28 days of lifitegrast 5\% treatment BID. The mean absolute difference in axial length after treatment with lifitegrast was $0.12 \pm 0.57 \mathrm{~mm}$. The mean absolute difference in steep keratometry values was $0.26 \pm 0.28 \mathrm{D}$ and $0.29 \pm 0.32 \mathrm{D}$ in flat keratometry values. The average absolute change in the meridian of the steep keratometric axis was $22.8 \pm 24.1^{\circ}$. Fifty-eight patients had manifest refraction data available 1 month after surgery. The accuracy of each preoperative biometry measurement was determined by comparing the predicted SE refraction for the lens actually implanted to the actual post-op SE measured at 1 month (Figure 1). In general, biometry performed after lifitegrast more closely predicted the final refractive outcome than with biometry performed on untreated dry eyes (prelifitegrast). Accuracy within $0.25 \mathrm{D}$ of the achieved SE was achieved in $47 \%$ of eyes before and $50 \%$ of eyes after lifitegrast. Within $0.5 \mathrm{D}$ of the achieved SE was predicted in $71 \%$ of measurements before and $79 \%$ after lifitegrast. Within $0.75 \mathrm{D}$ of the achieved SE was predicted by $81 \%$ of biometry measurements performed before and $91 \%$ after 


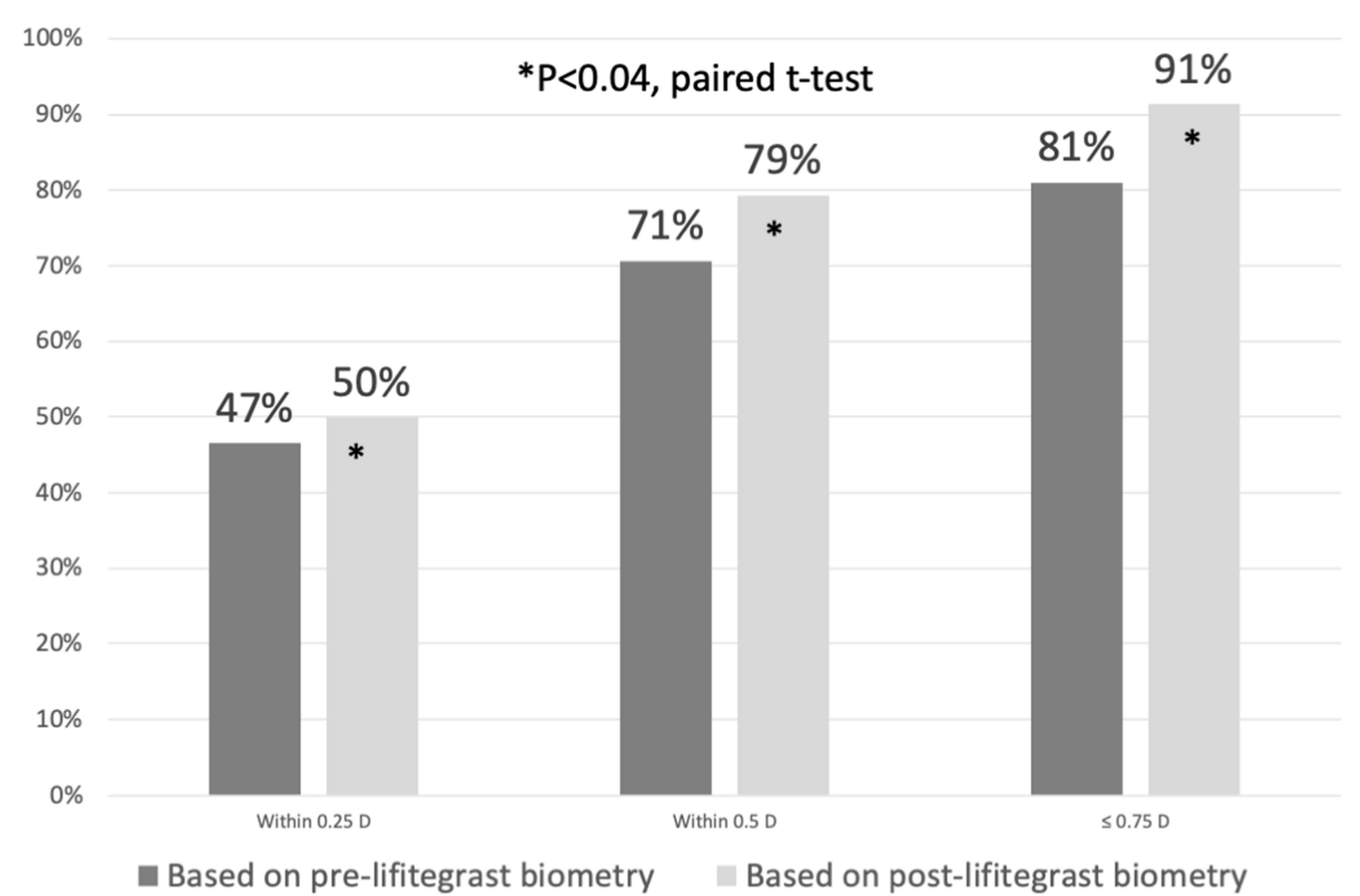

Figure I Preoperative biometry performed after 28 days of lifitegrast predicted the I month postoperative outcome with significantly greater accuracy than preoperative biometry measured before lifitegrast treatment.

lifitegrast. This improvement in biometry accuracy following lifitegrast was statistically significant $(\mathrm{P}<0.04$, paired $t$-test $)$.

A total of 77 patients were identified with preoperative higher than normal HOAs; 50 of those patients completed the full two courses of lifitegrast. Preoperatively, 43 patients $(56 \%)$ had a reduction in HOAs after being treated with the first course of lifitegrast (Figure 2). Considering the group as a whole, paired t-testing showed an overall statistically significant improvement (decrease) in HOAs from baseline to the preoperative measurements performed after lifitegrast $(\mathrm{P}$ $<0.001)$. Comparing baseline corneal HOAs to those measured after the post-surgery course of lifitegrast, HOAs

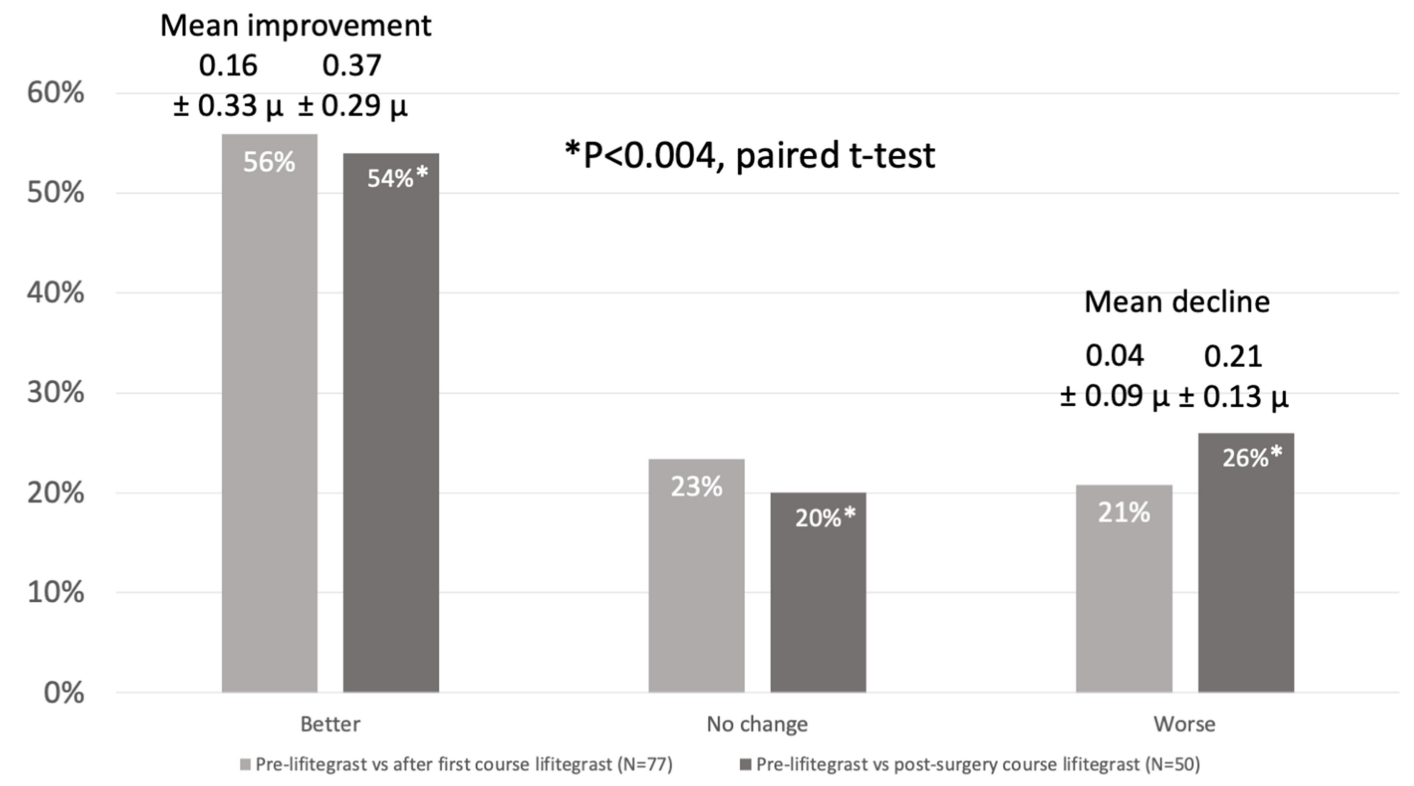

Figure 2 Preoperative total corneal higher order aberrations improved in a significant majority of patients treated with lifitegrast before surgery. 
improved in $27(54 \%)$ by a mean of $0.37 \pm 0.29 \mu$, remained the same in $10(20 \%)$, and increased in $13(26 \%)$ by a mean of $0.21 \pm 0.13 \mu$. Paired t-testing showed this to be a statistically significant improvement in HOAs $(\mathrm{P}<0.004)$.

Using the established $\mathrm{HOA} \leq 0.5 \mu$ threshold, of the 77 dry eyes studied with HOAs, $32 \%$ ( 25 eyes) were candidates for a multifocal lens before lifitegrast versus 52\% (40 eyes) after the first course of preoperative lifitegrast-a $60 \%$ increase in the pool of candidates for advanced technology.

\section{Secondary Outcomes}

The mean overall SPEED scores at baseline were $8.1 \pm$ 6.6, which improved to $6.3 \pm 4.9$ after the initial 28 days of lifitegrast and $4.0 \pm 3.2$ after surgery and the second round of lifitegrast $(\mathrm{P}<0.0004$, paired $t$-test, Table 1 and Figure 3). SPEED scores $>10$ are considered symptomatic of DED. ${ }^{36}$ Scores greater than 10 were reported at baseline by $30(29 \%)$ patients, after the first course by $11(13 \%)$ patients, and after the second course by one (2\%) patient.

Corneal staining (measured with the Oxford Scale) was present in all patients at baseline as an inclusion criterion, with $60(58 \%)$ having grade 1 stain, $40(39 \%)$ with grade 2 , and 3 patients $(3 \%)$ having grade 3 or higher stain. After the first course of lifitegrast, 52 (63\%) had no staining, 29 $(35 \%)$ had grade 1 , and 2 patients $(2 \%)$ had grade 2 or greater staining. After the second course of lifitegrast, corneal staining was not present in $27(54 \%)$, grade 1 in $21(42 \%)$, and grade 2 in $2(4 \%)$ of patients $(\mathrm{P}<0.00001$, paired $t$-test compared to baseline for both first and second courses of lifitegrast).

TBUT improved from a mean of $5.1 \pm 1.7$ seconds at baseline to $7.8 \pm 2.6$ after the first course of lifitegrast to $8.5 \pm 2.5$ seconds at the final assessment after the second course of lifitegrast ( $\mathrm{P}<0.0001$, paired $t$-test).

Conjunctival redness, measured with the Schulze Scale at baseline was grade 10 in 38 (37\%), grade 20 in 50 (49\%), and grade 30 or greater in $15(14 \%)$ of eyes. After the first course of lifitegrast, redness improved to grade 10 in $58(69 \%)$, grade 20 in $17(20 \%)$, and grade 30 or greater in $9(11 \%)$ of eyes. After the second course of lifitegrast, redness was grade 10 in $33(80 \%)$, grade 2 in 8 $(20 \%)$ and no eyes had greater than grade 30 redness. This was a statistically significant improvement in redness $(\mathrm{P}<$ 0.0001 for pre-treatment vs both post-treatment assessments, paired $t$-test).

\section{Discussion}

Our findings show that adding lifitegrast to treat preoperative dry eye (while allowing patients to maintain their current treatment regimens) leads to an improved accuracy of anterior corneal power measurements in predicting postoperative SE refractive error and, therefore, produces better postoperative outcomes. An unexpected finding was that lifitegrast also significantly reduced root-mean-square HOAs in the central $6.0 \mathrm{~mm}$ of the cornea. As expected, treatment with the dry eye drug also significantly reduced dry eye symptoms as measured by SPEED scores and significantly improved dry eye signs such as corneal staining, ocular redness, and TBUT. Although the improvement in dry eye signs and symptoms may not be novel or surprising in light of the OPUS-1, OPUS-2, and OPUS-3 trials establishing its efficacy, ${ }^{24,26-28}$ our study further confirms its clinical utility in this surgical setting. This study followed a number of patients for an additional month of lifitegrast that began \pm 28 days after surgery and continued until Day \pm 56 , and these patients retained a beneficial ocular surface effect of lifitegrast.

Our findings add to the literature by showing that lifitegrast has utility beyond improving DED signs and symptoms. ${ }^{23-29}$ To our knowledge, this is the first study demonstrating that lifitegrast can improve the refractive accuracy of preoperative anterior corneal power measurement readings while also reducing HOAs and decreasing dry eye signs and symptoms in cataract patients. To our knowledge, this is also the first study to show an improvement in the ocular surface when the drug was instituted

Table I Secondary Outcome Measures Showed Significant Improvement for All Measures at All Time Points

\begin{tabular}{|l|l|l|l|l|}
\hline & Baseline & $\begin{array}{l}\text { Preoperative After } \\
\text { Lifitegrast }\end{array}$ & $\begin{array}{l}\text { Postoperative Taking } \\
\text { Lifitegrast }\end{array}$ & $\begin{array}{l}\text { P value (Paired } \\
\text { t-Test) }\end{array}$ \\
\hline SPEED Score (mean \pm stdev) & $8.1 \pm 6.6$ & $6.3 \pm 4.9$ & $4.0 \pm 3.2$ & $<0.0004$ \\
Patients with grade I corneal staining & $60(58 \%)$ & $52(63 \%)$ & $21(42 \%)$ & $<0.0000$ I \\
TBUT (sec) & $5.1 \pm 1.7$ & $7.8 \pm 2.6$ & $8.5 \pm 2.5$ & $<0.0001$ \\
Patients with conjunctival erythema (Schulze & $65(63 \%)$ & $26(31 \%)$ & $8(20 \%)$ & $<0.0001$ \\
Scale) $\geq 20$ & & & & \\
\hline
\end{tabular}




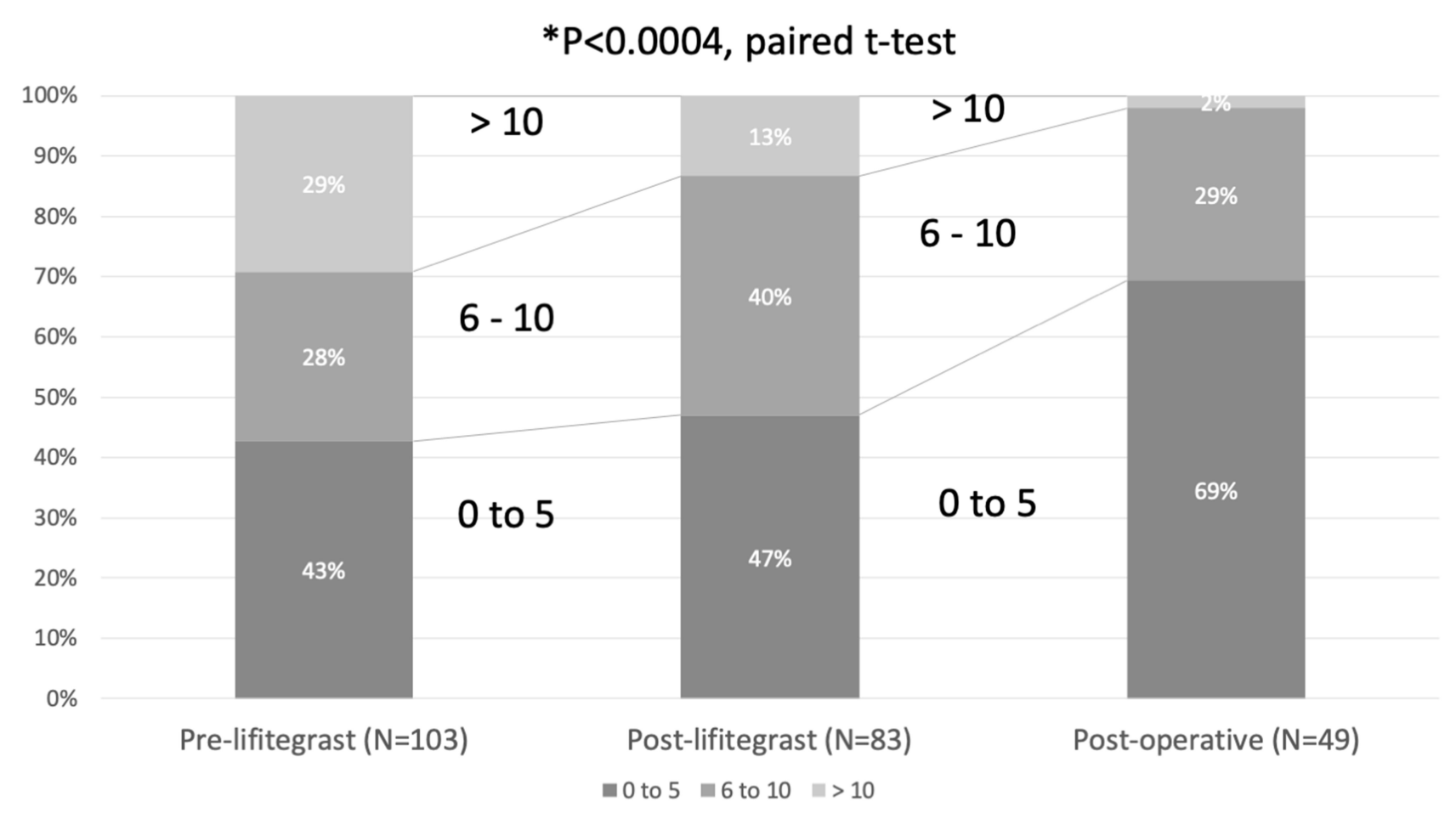

Figure 3 SPEED scores improved significantly after initial lifitegrast treatment and continued to improve following ongoing lifitegrast treatment postoperatively.

following a cataract patient's postoperative care. Because refractive accuracy and long-term improvement of the ocular surface has become essential to achieve patient satisfaction when refractive cataract surgery is performed, we encourage additional studies assessing other dry eye treatments before and after cataract surgery to continue to improve outcomes and increase patient satisfaction rates.

There are limitations to this study. We had a noncompliance rate with the first course of lifitegrast of almost $20 \%$ that is unexplained, as the drug was provided free of charge to patients. While we did not formally collect the reasons for discontinuation, anecdotal reports from patients suggested that discomfort with drop instillation was the chief reason. This is consistent with Donnenfeld et al reported that $54 \%$ of 220 patients experienced at least one treatmentemergent adverse event (AE) during the course of treatment with lifitegrast, with $12.3 \%$ discontinuing because of that AE. ${ }^{29}$ In that study, the most common AEs were instillation site irritation (burning), instillation site reaction, visual acuity reduced, dry eye, and dysgeusia. However, the OPUS-3 study found that only $3.4 \%$ of 293 patients in the lifitegrast arm reported any $\mathrm{AE}^{27}$ As in the Donnenfeld study, the most common AE in the OPUS study was site instillation burning and/or discomfort. ${ }^{27,29}$

Further, some of our patients did not return for their 1-month post-operative follow-up exam; however, our 58 patients were enough to generate a statistical significance threshold. Some patients did not receive a second round of treatment with lifitegrast after surgery; we did not capture data on the cause for declining a second course. However, about the first third of study participants completed the study before the additional month of lifitegrast (postoperatively) was added to the protocol. Loss to follow-up is a known issue in cataract patients, with one recent estimate from Gupta et al of about $15 \%{ }^{37}$ Factors leading to noncompliance are complex and not well understood, with a paucity of literature specifically examining the reasons behind loss to follow-up, which could range from overall patient dissatisfaction with the entire surgical process to dissatisfaction with the surgeon. On a more positive note, we hypothesize that if patients are satisfied with their surgical outcomes, they prefer to not return for additional follow-up visits or take additional drugs.

In this study, we sought to study only the effect of lifitegrast on surgical outcomes, so we did not instruct patients to use additional treatments with lifitegrast. In a routine clinical setting, we might try artificial tears, warm compresses, dietary supplements, or other steps to alleviate the patient's ocular surface issues. It is reasonable to assume these treatments might have also benefited patients, and it will be left for future study to determine how their efficacy might compare to, or be additive to, that 
of lifitegrast. To address that potential issue, when patients enrolled, we instructed them not to discontinue or change any baseline dry eye treatments they were using (artificial tears, etc.), so the results reported here can be attributed solely to the addition of lifitegrast. Based on our findings, we recommend assessing and optimizing the ocular surface before final biometry is performed in all cataract surgery candidates.

In conclusion, this study illustrates that cataract patients with dry eye can be treated with lifitegrast $5 \%$ before surgery to improve the optical quality of the anterior corneal tear film as evidenced by lowered corneal HOAs, which in turn increases the accuracy of predicting post-operative SE refractive error. Continuing lifitegrast after surgical provides a continued improvement in ocular surface quality compared to baseline.

\section{Data Sharing Statement}

The corresponding author will provide de-identified data to any legitimate investigator with a reasonable request for 5 years from the date of publication.

\section{Acknowledgments}

All authors are consultants to Novartis, which funded the study. Faith Hayden and Michelle Dalton, ELS, of Dalton \& Associates, Inc., provided medical writing assistance; this assistance was funded by MDBackline, LLC.

\section{Disclosure}

Dr John Hovanesian reports grants from and consultancy for Novartis, during the conduct of the study; grants from and consultancy for Novartis, outside the submitted work. Dr Alice Epitropoulos reports non-financial support from Novartis, during the conduct of the study; grants, nonfinancial support from Novartis Pharmaceuticals Corporation, outside the submitted work; and Consultant/ Speaker - Novartis. Dr Eric D Donnenfeld reports personal fees from Novartis, during the conduct of the study; personal fees from Allergan, outside the submitted work. The authors report no other potential conflicts of interest for this work.

\section{References}

1. Monestam E. Long-term outcomes of cataract surgery: 15-year results of a prospective study. J Cataract Refract Surg. 2016;42(1):19-26. doi:10.1016/j.jcrs.2015.07.040

2. Gollogly HE, Hodge DO, St Sauver JL, Erie JC. Increasing incidence of cataract surgery: population-based study. J Cataract Refract Surg. 2013;39(9):1383-1389. doi:10.1016/j.jcrs.2013.03.027
3. Lee AC, Qazi MA, Pepose JS. Biometry and intraocular lens power calculation. Curr Opin Ophthalmol. 2008;19(1):13-17. doi:10.1097/ ICU.0b013e3282f1c5ad

4. Jeong J, Song H, Lee JK, et al. The effect of ocular biometric factors on the accuracy of various IOL power calculation formulas. $B M C$ Ophthalmol. 2017;17(1):62. doi:10.1186/s12886-017-0454-y

5 . Norrby S. Sources of error in intraocular lens power calculation. $J$ Cataract Refract Surg. 2008;34(3):368-376. doi:10.1016/j. jcrs.2007.10.031

6. Sheard R. Optimising biometry for best outcomes in cataract surgery. Eye (Lond). 2014;28(2):118-125. doi:10.1038/eye.2013.248

7. Sahin A, Hamrah P. Clinically relevant biometry. Curr Opin Ophthalmol. 2012;23(1):47-53.

8. Jin GJ, Crandall AS, Jones JJ. Intraocular lens exchange due to incorrect lens power. Ophthalmology. 2007;114(3):417-424. doi:10.1016/j.ophtha.2006.07.041

9. Jin GJ, Merkley KH, Crandall AS, Jones YJ. Laser in situ keratomileusis versus lens-based surgery for correcting residual refractive error after cataract surgery. J Cataract Refract Surg. 2008;34 (4):562-569. doi:10.1016/j.jcrs.2007.11.040

10. Montes-Mico R. Role of the tear film in the optical quality of the human eye. J Cataract Refract Surg. 2007;33(9):1631-1635. doi:10.1016/j.jcrs.2007.06.019

11. Koh S, Maeda N, Hirohara Y, et al. Serial measurements of higher-order aberrations after blinking in patients with dry eye. Invest Ophthalmol Vis Sci. 2008;49(1):133-138. doi:10.1167/iovs.07-0762

12. Oh T, Jung Y, Chang D, et al. Changes in the tear film and ocular surface after cataract surgery. Jpn $J$ Ophthalmol. 2012;56 (2):113-118. doi:10.1007/s10384-012-0117-8

13. Movahedan A, Djalilian AR. Cataract surgery in the face of ocular surface disease. Curr Opin Ophthalmol. 2012;23(1):68-72. doi:10.1097/ICU.0b013e32834d90b7

14. Epitropoulos AT, Matossian C, Berdy GJ, et al. Effect of tear osmolarity on repeatability of keratometry for cataract surgery planning. $J$ Cataract Refract Surg. 2015;41(8):1672-1677. doi:10.1016/j. jers.2015.01.016

15. ASCRS. ASCRS Clinical Survey 2016. 2016.

16. ASCRS. ASCRS Clinical Survey 2015. 2015.

17. American Society of Cataract and Refractive Surgery. ASCRS Clinical Survey 2014. Global Trends in Ophthalmology. Fairfax, VA: American Society of Cataract and Refractive Surgery; 2014.

18. Trattler WB, Majmudar PA, Donnenfeld ED, et al. The Prospective Health Assessment of Cataract Patients' Ocular Surface (PHACO) study: the effect of dry eye. Clin Ophthalmol. 2017;11:1423-1430. doi:10.2147/OPTH.S120159

19. Yao K, Bao Y, Ye J, et al. Efficacy of $1 \%$ carboxymethylcellulose sodium for treating dry eye after phacoemulsification: results from a multicenter, open-label, randomized, controlled study. $B M C$ Ophthalmol. 2015;15:28. doi:10.1186/s12886-015-0005-3

20. Lee JH, Song IS, Kim KL, Yoon SY. Effectiveness and optical quality of topical $3.0 \%$ diquafosol versus $0.05 \%$ cyclosporine $\mathrm{A}$ in dry eye patients following cataract surgery. $J$ Ophthalmol. 2016;2016:8150757. doi:10.1155/2016/8150757

21. Devendra J, Singh S. Effect of oral lactoferrin on cataract surgery induced dry eye: a randomised controlled trial. J Clin Diagn Res. 2015;9(10):NC06-9.

22. Pflugfelder SC, Geerling G, Kinoshita S, et al. Management and therapy of dry eye disease: report of the management and therapy subcommittee of the international dry eye workshop (2007). Ocul Surf. 2007;5(2):163-178. doi:10.1016/S1542-0124(12)70085-X

23. Perez VL, Pflugfelder SC, Zhang S, et al. Lifitegrast, a novel integrin antagonist for treatment of dry eye disease. Ocul Surf. 2016;14 (2):207-215. doi:10.1016/j.jtos.2016.01.001

24. Keating GM. Lifitegrast ophthalmic solution 5\%: a review in dry eye disease. Drugs. 2017;77(2):201-208. doi:10.1007/s40265-0160681-1 
25. Xiidra FD. Prescribing Information. Lexington, MA: Shire; 2017.

26. Holland EJ, Luchs J, Karpecki PM, et al. Lifitegrast for the treatment of dry eye disease: results of a phase III, randomized, double-masked, placebo-controlled trial (OPUS-3). Ophthalmology. 2017;124 (1):53-60. doi:10.1016/j.ophtha.2016.09.025

27. Sheppard JD, Torkildsen GL, Lonsdale JD, et al. Lifitegrast ophthalmic solution $5.0 \%$ for treatment of dry eye disease: results of the OPUS-1 Phase 3 study. Ophthalmology. 2014;121(2):475-483. doi:10.1016/j.ophtha.2013.09.015

28. Tauber J, Karpecki P, Latkany R, et al. Lifitegrast ophthalmic solution $5.0 \%$ versus placebo for treatment of dry eye disease: results of the randomized phase III OPUS-2 study. Ophthalmology. 2015;122 (12):2423-2431. doi:10.1016/j.ophtha.2015.08.001

29. Donnenfeld ED, Karpecki PM, Majmudar PA, et al. Safety of lifitegrast ophthalmic solution $5.0 \%$ in patients with dry eye disease: a 1-year, multicenter, randomized, placebo-controlled study. Cornea. 2016;35(6):741-748. doi:10.1097/ICO.0000000000000803

30. Nichols KK, Donnenfeld ED, Karpecki PM, et al. Safety and tolerability of lifitegrast ophthalmic solution $5.0 \%$ : pooled analysis of five randomized controlled trials in dry eye disease. Eur J Ophthalmol. 2019;29(4):394-401. doi:10.1177/1120672118791936
31. Donnenfeld ED, Perry HD, Nattis AS, Rosenberg ED. Lifitegrast for the treatment of dry eye disease in adults. Expert Opin Pharmacother. 2017;18(14):1517-1524. doi:10.1080/14656566.2017.1372748

32. McCormick GJ, Porter J, Cox IG, MacRae S. Higher-order aberrations in eyes with irregular corneas after laser refractive surgery. Ophthalmology. 2005;112(10):1699-1709. doi:10.1016/j.ophtha.2005.04.022

33. Mirzajani A, Aghataheri S, Ghoreishi M, et al. Evaluation of corneal higher order aberrations in normal topographic patterns. J Curr Ophthalmol. 2016;28(2):75-80. doi:10.1016/j.joco.2016.03.001

34. Schulze MM, Jones DA, Simpson TL. The development of validated bulbar redness grading scales. Optom Vis Sci. 2007;84(10):976-983. doi:10.1097/OPX.0b013e318157ac9e

35. Simpson TL, Situ P, Jones LW, Fonn D. Dry eye symptoms assessed by four questionnaires. Optom Vis Sci. 2008;85(8):E692-9. doi:10.1097/OPX.0b013e318181ae36

36. Blackie CA, Solomon JD, Scaffidi RC, et al. The relationship between dry eye symptoms and lipid layer thickness. Cornea. 2009;28(7):789-794. doi:10.1097/ICO.0b013e318191b870

37. Gupta S, Ravindran RD, Subburaman GB, et al. Predictors of patient compliance with follow-up visits after cataract surgery. J Cataract Refract Surg. 2019;45(8):1105-1112. doi:10.1016/j.jcrs.2019.02.024
Clinical Ophthalmology

\section{Publish your work in this journal}

Clinical Ophthalmology is an international, peer-reviewed journal covering all subspecialties within ophthalmology. Key topics include: Optometry; Visual science; Pharmacology and drug therapy in eye diseases; Basic Sciences; Primary and Secondary eye care; Patient Safety and Quality of Care Improvements. This journal is indexed on PubMed

\section{Dovepress}

Central and CAS, and is the official journal of The Society of Clinical Ophthalmology (SCO). The manuscript management system is completely online and includes a very quick and fair peer-review system, which is all easy to use. Visit http://www.dovepress.com/ testimonials.php to read real quotes from published authors. 